\title{
Designing molecular terahertz generation crystals: optimal packing could be just one carbon away
}

Gabriel A. Valdivia-Berroeta, Adam X. Wayment, Karissa C. Kenney, Erika W. Jackson, Isaac C. Tangen, Charles B. Bahr, Stacey J. Smith, David J. Michaelis, Jeremy A. Johnson.

Department of Chemistry and Biochemistry, Brigham Young University, Provo, UT, 84602, USA

Organic nonlinear optical materials are used for a wide range of applications, including terahertz $(\mathrm{THz})$ and second harmonic generation. Optimal molecular arrangements in the crystal structure are judged by $\theta_{p}$, which refers to the angle between the main transfer axis and the crystallographic axis. $\theta_{p}$ ranges from $0^{\circ}$ for perfectly aligned noncentrosymmetric packing to $90^{\circ}$ for centrosymmetric arrangement. Because noncentrosymmetric packing is essential to obtain a second-order material response, several strategies have been designed to overcome the anti-parallel arrangements often favored by dipole-dipole interactions. One of the most common approaches involves inducing hydrogen-bonding networks between chromophores. However, hydroxyl groups decrease the molecular hyperpolarizability compared to N-alkyl and O-alkyl groups, such as -OMe or - $\mathrm{N}\left(\mathrm{Me}_{2}\right)$. A different method takes advantage of the ability of organic salts to pair the same THz-generating cation with different anions introducing different interactions and thereby changing the molecular packing from centrosymmetric to noncentrosymmetric. Here, we introduce an additional means of controlling crystal structure: cation modification. By switching a methyl (1-carbon) for an ethyl (2-carbon) group in the quaternary nitrogen moiety of the cation, we observe dramatic changes in $\theta_{p}$ from $90^{\circ}$ to $0^{\circ}$ without significantly impacting hyperpolarizability. We attribute this packing behavior to the increased steric effect of the ethyl group that results in larger intermolecular distances and so enables a parallel, noncentrosymmetric arrangement of chromophores in the crystalline state. This engineering strategy will deeply impact the way crystals for nonlinear optical applications are designed.

Contact Information:

Gabriel Valdivia gvaldivia@byu.net

Adam Wayment adam.wayment@duke.edu

Karissa Kenney karissakenney@gmail.com

Erika Jackson erika.weir@hotmail.com

Isaac Tangen tangen.isaac@gmail.com

Charles Bahr charlie.bahr@gmail.com

Stacey Smith ssmith@chem.byu.edu

David Michaelis dmichaelis@chem.byu.edu

Jeremy Johnson jjohnson@chem.byu.edu 